\title{
Application of Shape Analysis on renal tumors in 3D
}

\author{
Stefan Giebel, Jang Schiltz, and Jens-Peter Schenk
}

Index Terms-Shape, Mean Shape Renal tumors

Abstract-There are different kinds of tumours in childhood: nephroblastoma, clear cell sarcoma, neuroblastoma etc. For diagnosis MRI (Magnetic resonance images) are used. Our research is the first mathematical approach on $\mathrm{MRI}$ (Magnetic resonance images) of renal tumours. We are using transversal, frontal and sagittal images and compare them in their potential for differentiation different kind of tumours. The procedure of getting three dimensional landmarks by using the edges of the platonic body (C60) as well as a test for the mean shape with the same diagnosis to all others are shown. Eventually we discuss the results for the oncology.

\section{INTRODUCTION}

In a wide variety of disciplines it is of great practical importance to measure, to describe and compare the shapes of objects. In general terms, the shape of an object, data set, or image can be defined as the total of all information that is invariant under translations, rotation and isotropic rescaling. The field of shape analysis involves hence methods for the study of the shape of objects where location, rotation and scale can be removed. The twoor more dimensional objects are summarized according to key points called landmarks. This approach provides an objective methodology for classification whereas even today in many applications the decision for classifying according to the appearance seems at most intuitive.

Statistical shape analysis is concerned with methodology for analyzing shapes in the presence of randomness. It is a mathematical procedure to get the information of two- or more dimensional objects with a possible correction of size and position of the object. So objects with different size and/or position can be compared with each other and classified. To get the shape of an object without information about position and size, centralisation and standardisation procedures are used in some metric space.

Interest in shape analysis began in 1977. D.G. Kendall[14] published a note in which he introduced a new representation of shapes as elements of complex projective spaces. K.V. Mardia[17] on the other hand investigated the distribution of the shapes of triangles generated by certain point processes, and in particular considered whether towns in a plain are spread regularly with equal distances between neighbouring towns. The full details of this elegant theory which contains interesting areas of research for both probabilists and statisticians where published by D. Kendall[15] and F. Bookstein[2]. The details of the theory and further developments can be found in the textbooks by C.G. Small[20] and I.L. Dryden \& K.V. Mardia[10].

In this paper, we describe one interesting application of statistical shape analysis: the classification of renal tumors in childhood. In contrast to many application called also "Shape Analysis"[4] we have to determine a mean shape, representative for a group of objects, and not only to detect an already known shape. In our first approach to shape analysis we use Euclidean distance, but in many cases we know, that we have also to reflect on non-Euclidean transformations: The renal tumor is limited by spleen or liver, the rest of the kidney, the spine and retroperitoneal vessels. In Giebel(2007)[7] it 
was shown that every landmark has another meaning for differentiating the tumors. Only for the influence of the landmarks on the general shape there are no results according to the test of Ziezold (2003)[23] in Giebel, Schiltz, Schenk 2009[8]. Related is the work to the research of Bookstein 1996[3] on Brain MR scans of schizophrenic patients

\section{WILMS-TUMORS}

In the special case of oncology there is no theoretical medical reason to select a specific group of landmarks for differentiation. All landmarks in this research have thus to be selected by an explorative procedure.

Nephroblastoma (Wilms' tumor)[21] is the typical tumor of the kidneys appearing in childhood. Therapy is organized in therapyoptimizing studies of the Society of Paediatric Oncology and Haematology (SIOP). Indication of preoperative chemotherapy is based on radiological findings. The preferred radiological method is sonography and MRI. Both methods avoid radiation exposure, which is of great importance in childhood. Preoperative chemotherapy is performed without prior biopsy[18].

Information of the images of magnetic resonance tomography, especially the renal origin of a tumor and the mass effect with displacement of other organs, is needed for diagnosis. Next to nephroblastomas other tumors of the retro peritoneum exist, which are difficult to differentiate [19]. Renal tumors in childhood are classified in three stages of malignancy (I, II, III). Typical Wilms tumors mostly belong in stage II. In stage II different subtypes of nephroblastoma tissue exist[13].

In our sample of tumors in childhood,there are four different types of retroperitoneal tumours: nephroblastoma, neurobblastoma, clear cell carcinoma, and renal cell carcinoma. Renal cell carcinomas are very rare in childhood. They represent the typical tumors of adult patients. They have no high sensitivity for chemotherapy. Clear cell sarcomas are very rare in childhood and are characterized by high malignancy. Neuroblastoma is the main

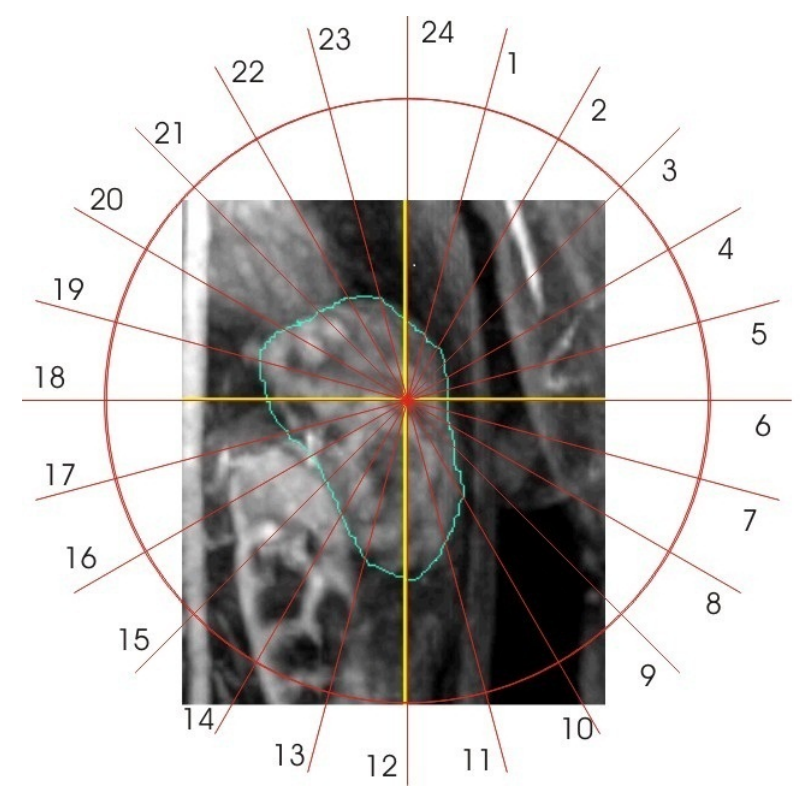

Fig. 1: 2D-image of the tumour

differential diagnosis to nephroblastoma. It is the typical tumor of the sympathetic nervous system and suprarenal glands. Infiltration of the kidney is possible. The tumor grows with encasement of vessels. Because of the high importance of radiological diagnosis for therapy, it is of great interest to find markers for a good differentiation of tumors. MRI produces 2D-images. From the two dimensional data a three dimensional object has to be approximated in each case. In the image 1 the raw data are shown:

\section{Mean Shape}

To compare the standardized and centered sets of landmarks, we have to define the mean shape of all the objects and a distance function which allows us to evaluate how "near" every object is from this mean shape.

The term "mean" is here used in the sense of Fréchet (1948)[12]. If $X$ denotes a random variable defined on a probability space $(\Omega, \mathcal{F}, \mathcal{P})$ with values in a metric space $(\Xi, d)$, an element $m \in \Xi$ is called a mean of $x_{1}, x_{2}, \ldots, x_{k} \in \Xi$ if

$$
\sum_{j=1}^{k} d\left(x_{j}, m\right)^{2}=\inf _{\alpha \in \Xi} \sum_{j=1}^{k} d\left(x_{j}, \alpha\right)^{2} .
$$


That means that the "mean shape" is defined as the shape with the smallest variance of all shapes in a group of objects. For computing the mean shape we use the algorithm of Ziezold (1994)[22].

\section{Test of the MEAN SHAPE}

The test of Ziezold (1994)[22] can be described in following steps:

\section{1.step Definition of the set of objects}

There is one set $M=\left\{o_{1}, \ldots, o_{N}\right\}$ that can be divided into two subsets: objects with the characteristics A:

$A^{\text {sample }}=\left\{o_{1}, \ldots, o_{n}\right\}=\left\{a_{1}, \ldots, a_{n}\right\}$

and objects with the characteristics $B$ :

$B^{\text {sample }}=\left\{o_{n+1}, \ldots, o_{N}\right\}=\left\{b_{1}, \ldots, b_{N-n}\right\}$.

The subset $\mathrm{A}$ is a realisation of a distribution $P$ and the subset $\mathrm{B}$ is an independent realisation of a distribution $Q$.

Hypothesis: $\quad H_{0}: P=Q$

Alternative: $\quad H_{1}: P \neq Q$

Define the level of significance $\alpha$. If the probability for $H_{0}$ is smaller, we neglect $H_{0}$ and assume $H_{1}$.

\section{2. step: Computing the mean shape}

The mean shape is calculated by means of the algorithm of Ziezold (1994)[22]. Let $m_{0}$ denote the mean shape of the subset $A$.

3. step: Computing the $u$-value

$$
u_{0}=\sum_{j=1}^{n} \operatorname{card}\left(b_{k}: d\left(b_{k}, m_{0}\right)<d\left(a_{j}, m_{0}\right)\right)
$$

4. step: Determination of all the possibilities of dividing the set into two subset with the same proportion

5. step: Comparing the $u_{0}$-value to all possible $u$-values. Computing the rank (small uvalue mean a small rank).
6. step: Calculate the $p$-value for $H_{0}$

$p_{r=i}=\frac{1}{n_{\text {sample }}}$ for $i=1, \ldots, n_{\text {sample }}$, where $r$ is the rank for which we assume a rectangular distribution and $n_{\text {sample }}$ a randomized sample of all possibilities.

The same results are there after obtained also for the subset $B$.

\section{Results}

To get $3 D$ landmarks we construct from $2 D$-images a three dimensional object of the tumor. Then we take as landmarks the cutpoints between the surface of the tumor and the vector of the edge of the platonic body C60.

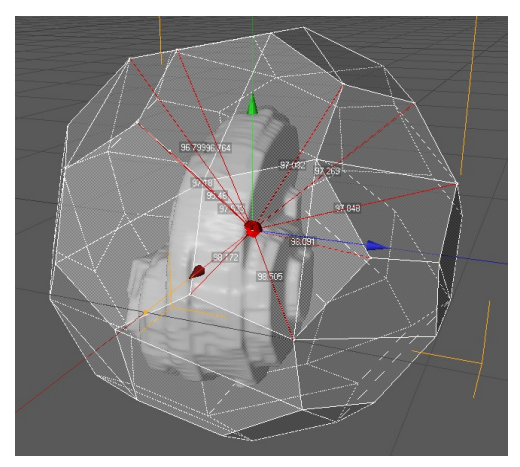

Fig. 2: 3D-Landmarks as cut points between the edge of a platonic body and the surface of the tumor

For a sample of 74 comparable tumors (69 nephroblastoma and 5 neuroblastoma) we get for the test of Ziezold[22] an $u_{0}$-value of 72 in the direction neuroblastoma vs. nephroblastoma. The $p$-value is according to the rank in a randomized sample $(n=1000) 0.116$. In the other direction nephroblastoma vs. neuroblastoma we get an $u_{0}$ value of 112 . According to the rank the $p$-value is 0.080 in a randomized sample. In the following image the mean shape of the nephroblastoma (Red) and of the neuroblastoma (Green) are shown.

\section{Conclusion}

There is a tendency for differentiating the neuroblastoma from the nephroblastoma by the 
"mean shape", especially by the "mean shape" of nephroblastoma. Shape Analysis is useful to make a decision inspite of different size, location etc. The test used for differentiating the different kind of tumors does not need any assumptions in regard to distributions and the size of the sample.

For improving our results the non-Euclidean transformations have to be reflected in the future. A possible approach is to use a supervised 1-layer neural network with weighted landmarks. Instead of the mistake between output and reality we will take the distance between the "mean shape" and the objects.

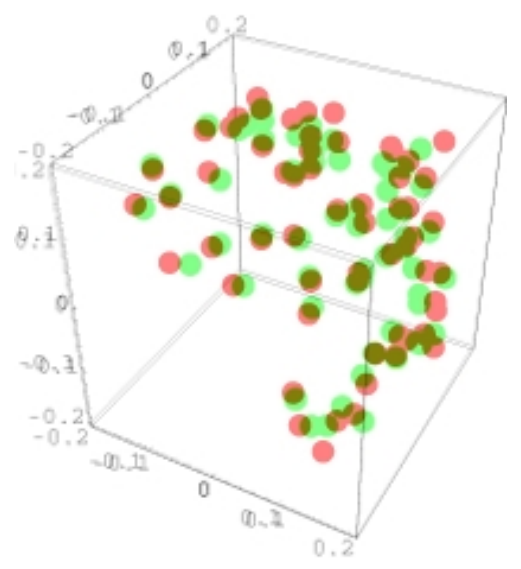

Fig. 3: Mean Shape: Red: 60 landmarks of the mean shape of the nephroblastoma, Green: 60 landmarks of the mean shape of the neuroblastoma

\section{RefEREnCES}

[1] Bishop, C.M., 1995, Neural networks for pattern recognition, Clarendon Press, Oxford.

[2] Bookstein, F.L., 1986, Size and shape spaces for landmark data in two dimensions, Statistic Sciences 1; p..181-242.

[3] Bookstein, F.L., 1996, Biometrics, biomathematics and the morphometric synthesis, Bulletin of Mathematical Biology 58; p.313-365.

[4] Favoro, Paolo, and Soatto, Stefano, 2007, 3D-Shape Estimation and Image Restoration, Springer, Berlin.

[5] Frechen, F.B., Franke, W. and Giebel, S., 2009, Biofiltration: From past to future - scientific view- Biotechniques for Air Pollution Control, avaliable on http://hrz-vm162.hrz.unikassel.de/web/SiwawiDokumente/pdf/publikationen.

[6] Giebel, S., 2007, Analyse of the spatio-temporal patterns of serial murderers (Analyse des räumlichen Verhaltens von Serienmördern), 3rd Statstical days, avaliable on http:/ / sma.uni.lu/stat3/files/Giebel2.pdf.
[7] Giebel, S., 2007, Statistical Analysis of the shape of renal tumors in childhood, Diploma thesis, University Kassel.

[8] Giebel, S., Schiltz, J., and Schenk, J.P., 2009,Application of Statistical Shape Analysis to the Classification of three dimensional renal tumors appearing in early childhood; avaliable on http://www3.iam.metu.edu.tr/juergenlehn/giebel.pdf

[9] Coppes, M.J., Campbell C.E., Williams, B.R.G., 1995, Wilms Tumor: Clinical and Molecular Characterization, Austin Texas USA: RG Landes Company,p.1-55

[10] Dryden, I. L; Mardia, K. V., 1998, Statistical Shape Analysis. Wiley, Chichester

[11] Furtwängler, R., Schenk, J.P., Graf, N. u.a., 2005, Nephroblastom- Wilms- Tumor, Onkologie 11.

[12] Fréchet, M.,1948,Random elements in the metric space (Les élements aléatoires de nature quelconque dans un espace distancié), Annales de l'Institut Henri Poincaré, Vol. 10, pp.215-310

[13] Graf, N., 2003, Urologe A 43:421

[14] Kendall, D.G., 1977, The diffusion of shape, Adv. Appl. Probab. 9, 1977

[15] Kendall, D.G., 1984, Shape manifolds, Procrustrean metrics and complex projective spaces, Bulletin of the London Mathematical Society, 16, p.81-121.

[16] Kendall D.G., Kendall W.S., 1980, Alignment in two dimensional random sets of points, Advances in Applied probability 12, p.380-424.

[17] Mardia, K.V., 1977, Mahalanobis distance and angles. In: Krishnaiah, P.R. (ed) Multivariate Analysis IV, p.495-511, Amsterdam: North Holland.

[18] Schenk, J.P, Fortschr. Röntgenstr. 178:38

[19] Schenk, J.P, 2008, Eur. Radiol. 18:683, 2008

[20] Small, C. G., 1996 The Statistical Theory of Shape. Springer-Verlag, New York.

[21] Wilms, M., 1889, Renal tumors (Die Mischgeschwülste der Niere), Verlag von Arthur Georgi, Leipzig, p.1-90.

[22] Ziezold, H., 1994, Mean Figures and Mean Shapes Applied to Biological Figure and Shape Distributions in the Plane, Biometrical Journal, 36, p. 491-510.

[23] Ziezold, H., 2003, Independence of Landmarks of Shapes. Mathematische Schriften Kassel, Heft 03
Stefan Giebel Stefan Giebel recieved the Master in sociology, a pre-degree in social pedagogy, a diploma in mathematics and the Ph.D. degree in sociology all from the university of Kassel in 2000, 2007 and 2009. He works on the relapse of juvenile offenders, on detection of drugs and explosive materials by using electronic noses etc. Since 2007 he has started his Ph.D. studies in mathematics in the university of Luxembourg about "Applying shape analysis on renal tumors". 